\title{
ACCURACy: Adaptive Calibration Of Cubesat Radiometer Constellations
}

\author{
John Bradburn, Mustafa Aksoy, Henry Ashley, \\ University at Albany, SUNY
}

\section{Presentation Outline}

Introduction

Technical Approach \& Methodology.. 


\section{Introduction}

- Current State of the Art methods for the intercalibration of constellations of radiometers are not sufficient for frequent recalibration of CubeSats which rely on vicarious calibration measurements.

*Futura-Sciences. "Miras." Futura-Sciences

www.futura-sciences.us/dico/d/astronautics-miras-

50005978/

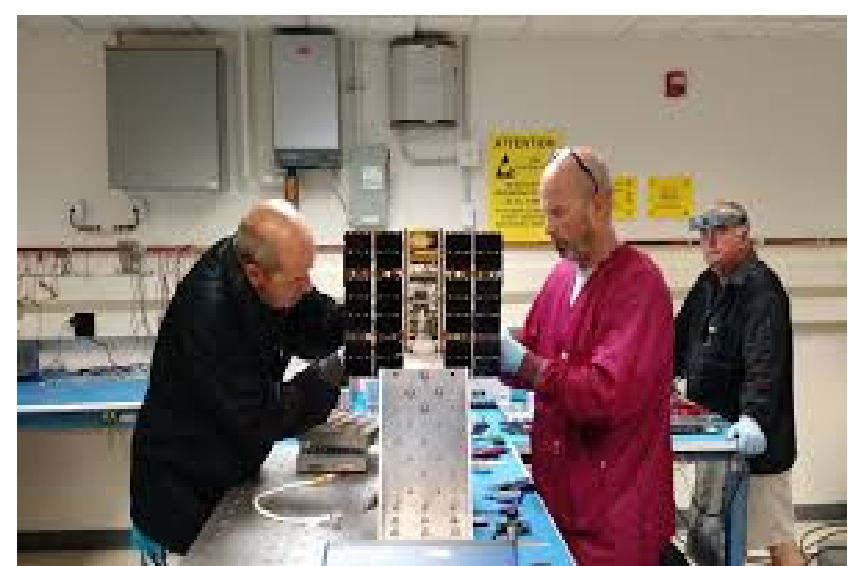

- We propose a novel, constellation-level calibration framework "Adaptive Calibration of Cubesat Radiometer Constellations", or ACCURACy. The aim of this framework is to develop a precise methodology for the intercalibration of a constellation of identical CubeSat radiometers, enabling a traceable, quantifiable, real-time, system-level absolute calibration. 


\section{Goals}

- Establish ML algorithm to gather radiometers in similar states into time-adaptive clusters

- Establish a procedure to form calibration data pools for each cluster such that calibration measurements from one cluster member may be shared in real-time with other cluster members

- Create a cluster-level $N>2$ point absolute calibration structure for each cluster using pooled calibration data

- Mathematically quantify errors and uncertainties in calibrated constellation measurements and study data processing and transmission requirements for scalability

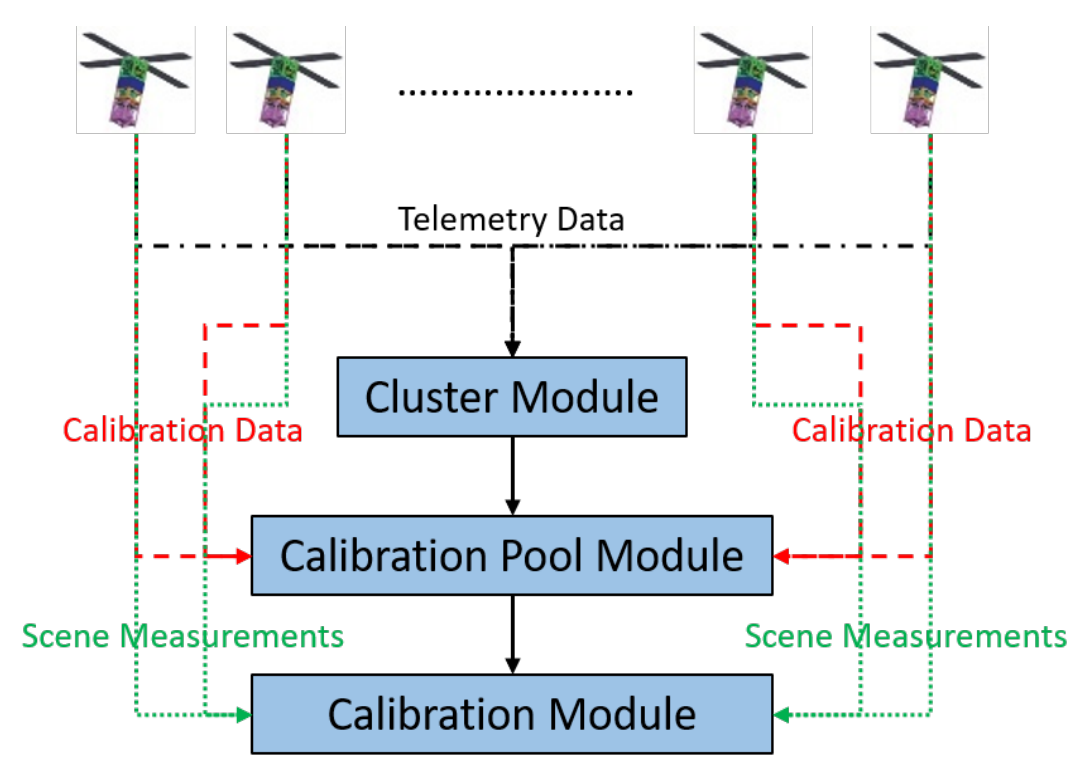

Overview of the ACCURACY Framework with Its Three Modules. 


\section{ACCURACy Framework}

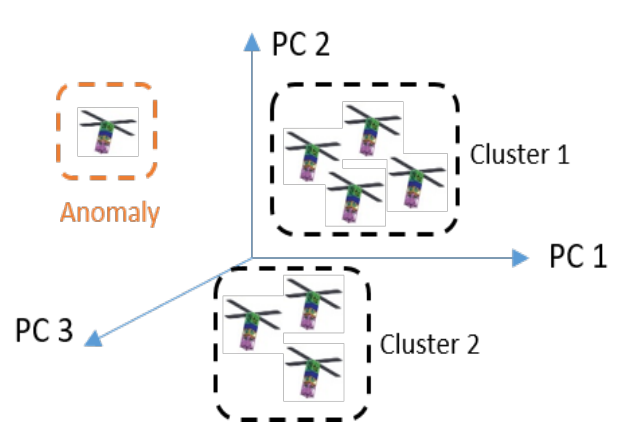

\section{Cluster Module}

Objective:

- Process telemetry data

- Classify radiometers in realtime

Implement:

- PCA - Dimensionality reduction

- Clustering

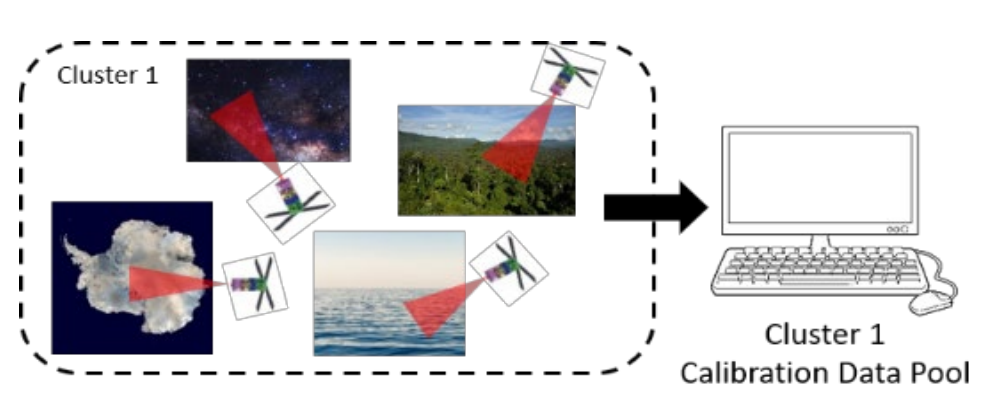

\section{Calibration Pool Module}

Objective:

- Collect real-time cluster information, calibration measurements and times

Implement:

- Data fusion and distribution

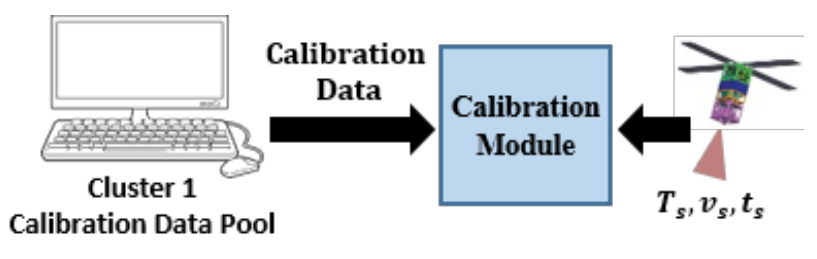

\section{Calibration Module}

Objective:

- Calibrate CubeSat radiometer using pooled calibration data

Implement:

- $\mathrm{N}>2$-point LLSE calibration

- Meet uncertainty and accuracy requirements for calibrated antenna 


\section{ACCURACy Framework}

\section{Cluster Module}

\section{Objective:}

- Process telemetry data

- Classify radiometers in realtime

\section{Implement:}

- PCA - Dimensionality reduction

- Clustering

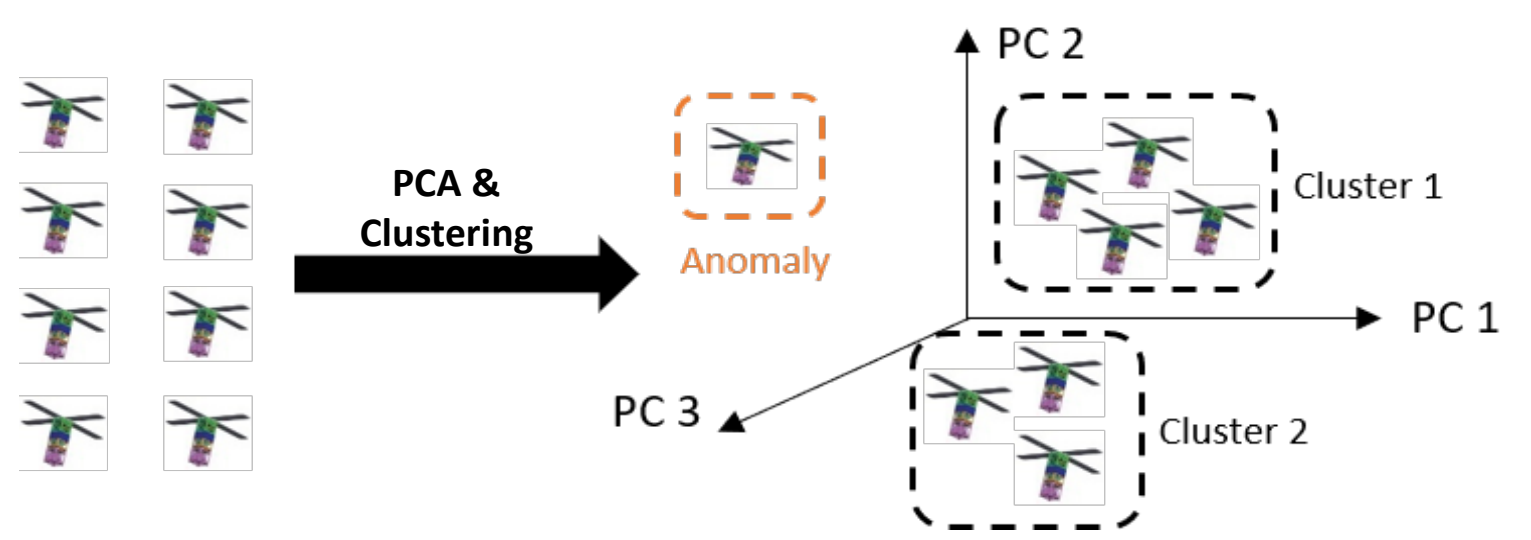

\section{Parameters:}

- Number of principal components

- Cluster size

- Anomaly criteria/health parameters

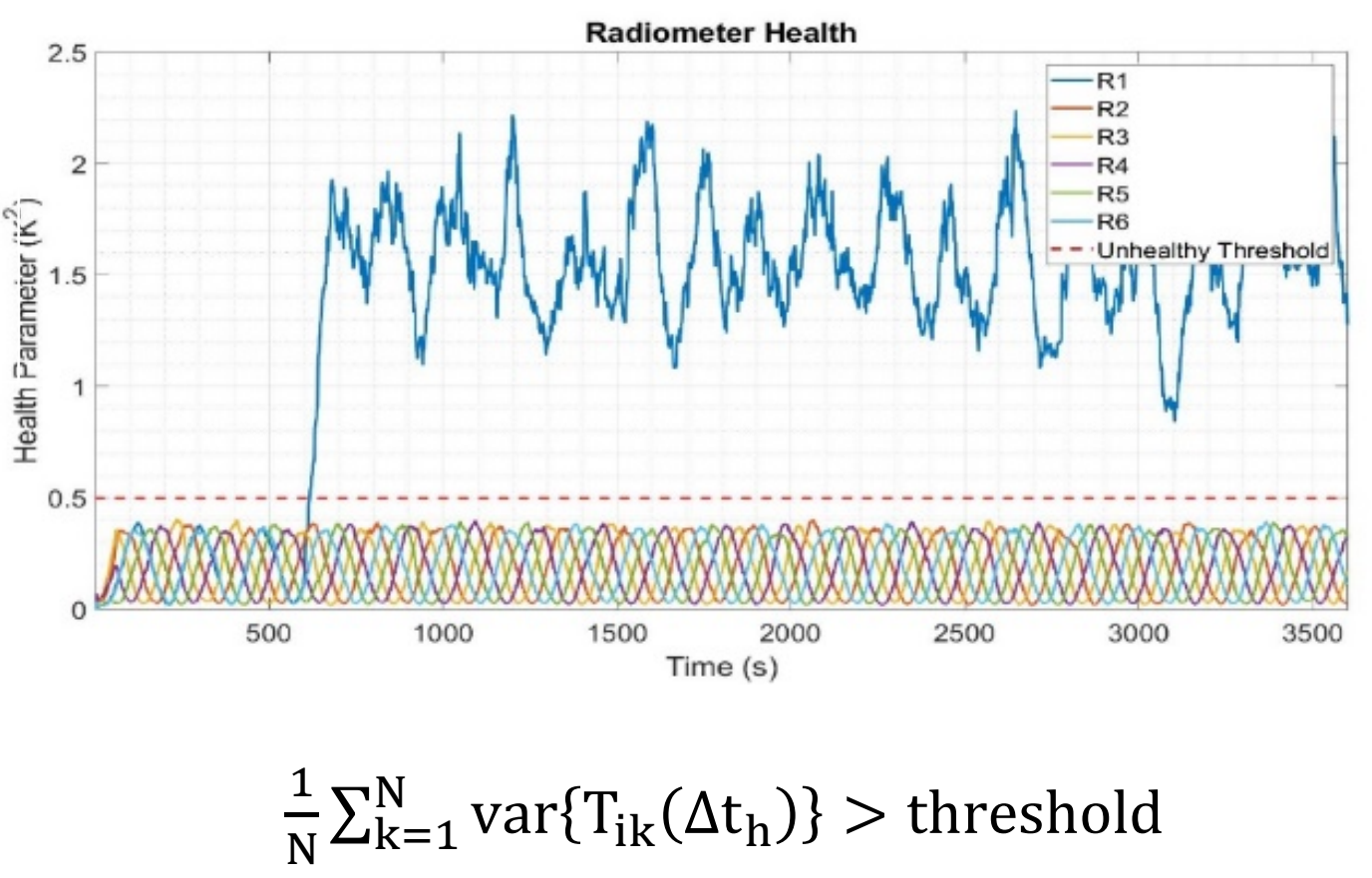

$$
\frac{1}{\mathrm{~N}} \sum_{\mathrm{k}=1}^{\mathrm{N}} \operatorname{var}\left\{\mathrm{T}_{\mathrm{ik}}\left(\Delta \mathrm{t}_{\mathrm{h}}\right)\right\}>\text { threshold }
$$

\section{Output:}

- Real-time cluster information for all radiometers

- N-dimensional telemetry data from all radiometers 


\section{ACCURACy Framework}

\section{Cluster Module}

Radiometer gain is usually characterized by the physical temperature of the system, and the age of the instrument.

The ACCURACY Cluster Module uses this relationship between the radiometer gain, physical temperature, and instrument age to determine clusters of constellation members in similar states.
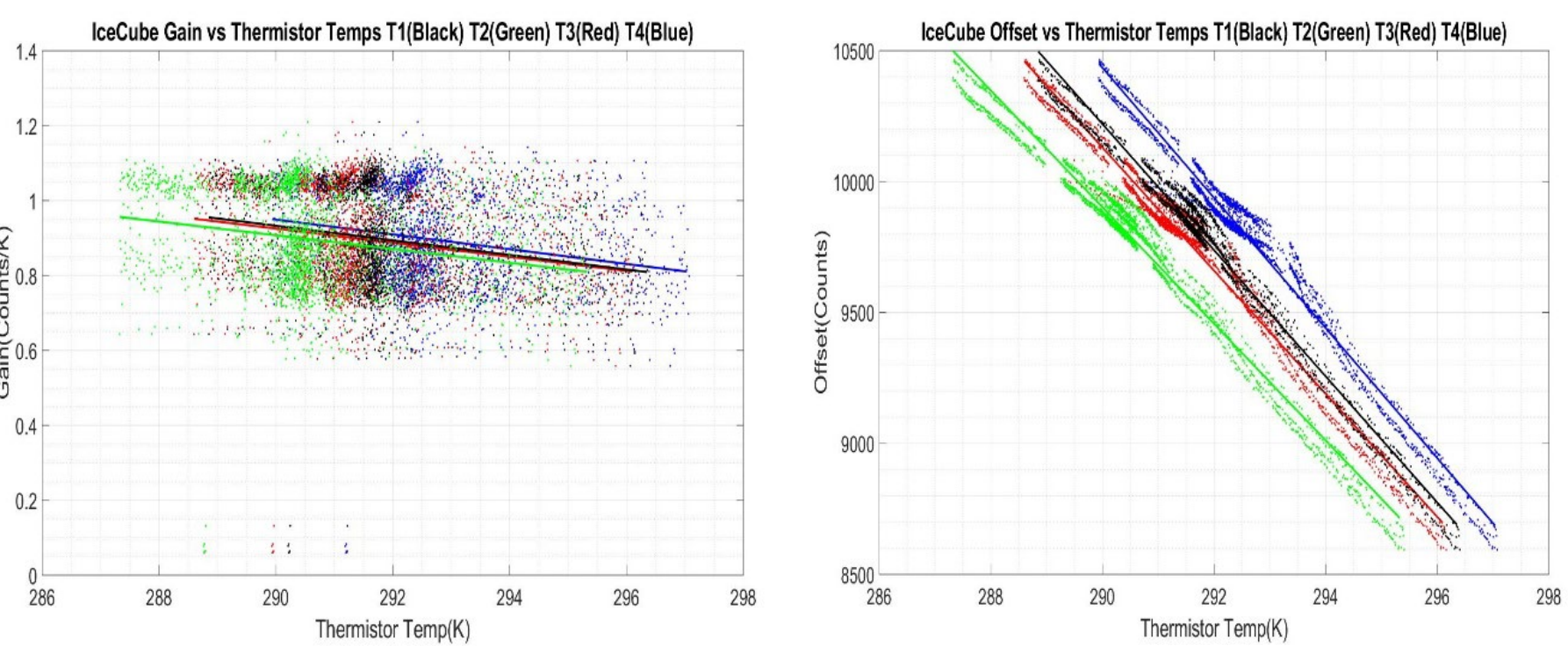

*Dong Wu, IceCube: Submm-Wave Technology Development for Future Science on a CubeSat
$M J J A S O N D J F M A M J J A S O N$

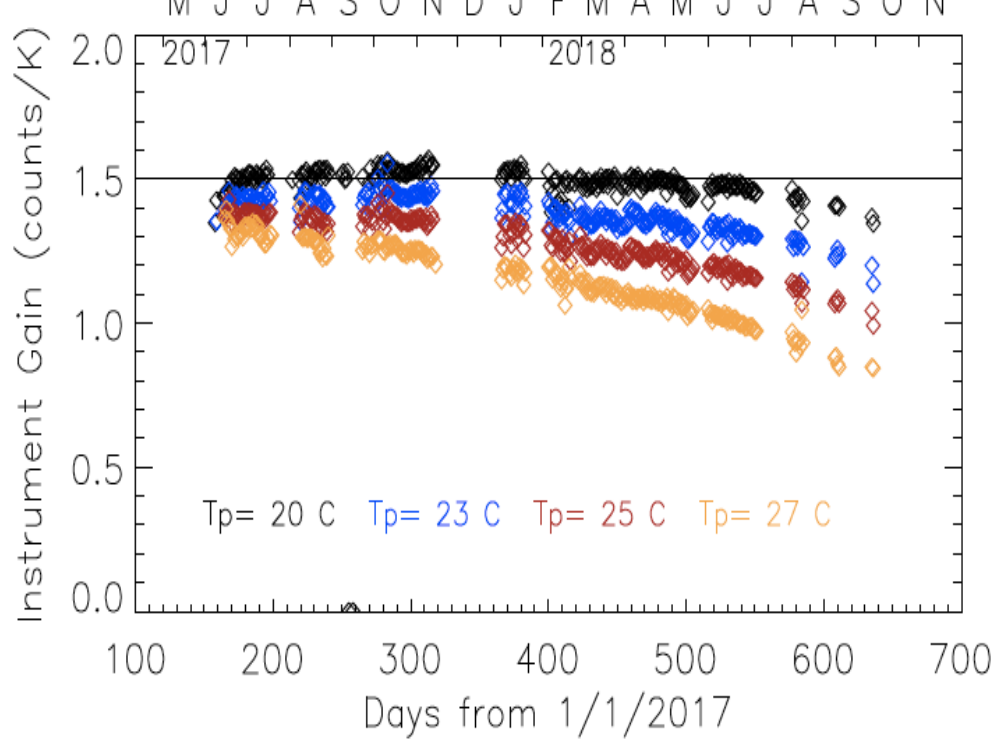

Gain of the $i^{\text {th }}$ radiometer in the constellation:

$$
g_{i}(t)=f\left(T_{i 1}(t), \ldots, T_{i N}(t), t_{i}\right)
$$

where $t_{i}$ is the age and $T_{i N}$ is the $N^{\text {th }}$ thermistor temperature of the $i^{\text {th }}$ radiometer. It is modeled as:

$$
g_{i}(t)=\sum_{k=1}^{N} W_{i k} T_{i k}(t)+W_{i t} t_{i}
$$




\section{ACCURACy Framework}

\section{Calibration Pool Module}

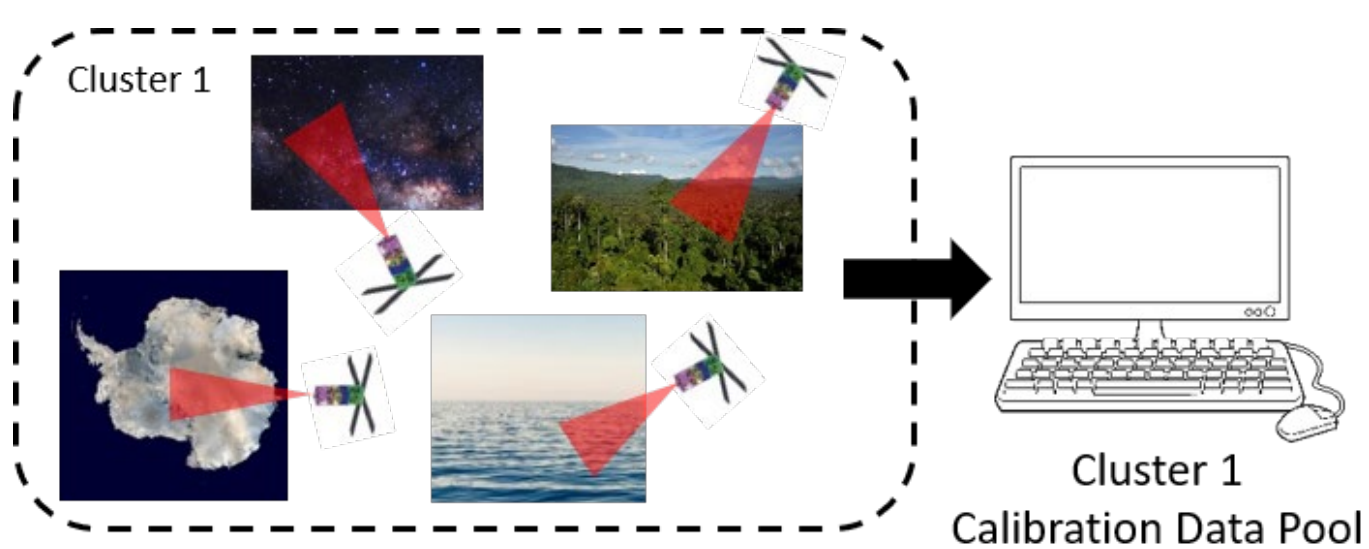

The Calibration Pool Module operating on a cluster with 4 radiometers. Vicarious calibration measurements and measurement times are collected in a data pool shared by all group members.

\section{Adjustable Parameters:}

- Availability time interval for individual calibration measurements

- How long calibration data stays "valid" within calibration data pools

\section{Objective:}

- Collect real-time cluster information, calibration measurements and times

\section{Implement:}

- Data fusion and distribution

\section{Input:}

- Real-time cluster information for all radiometers

- Calibration measurements and times

\section{Output:}

- Cluster-specific datasets containing pooled calibration data associated with radiometers in respective clusters 


\section{ACCURACy Framework}

\section{Calibration Module}

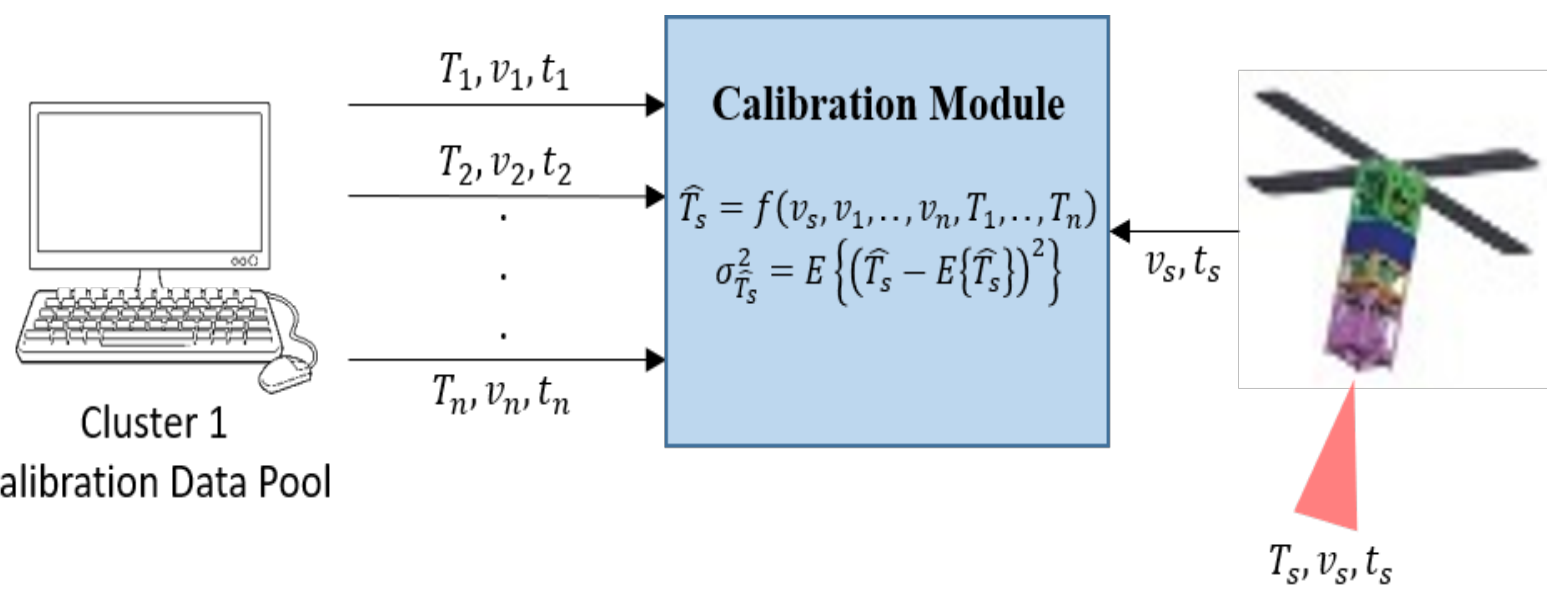

The Calibration Module calibrating a CubeSat radiometer in similar-state radiometer cluster using the associated calibration data pool.

\section{Adjustable Parameters:}

- Which calibration measurements to use in the calibration

- Uncertainty and accuracy requirements of calibrated products

\section{Objective:}

- Calibrate CubeSat radiometer using pooled calibration data

Implement:

- N>2-point LLSE calibration

- Meet uncertainty and accuracy requirements for calibrated antenna

Input:

- Calibration measurements and times from calibration pools for each cluster

- Scene measurements and associated times

\section{Output:}

- Calibrated scene measurements and associated times 


\section{ACCURACy Framework}

- The ACCURACy framework has been developed in MATLAB along with a radiometer simulator allowing the simulation of a constellation of identical CubeSat radiometers in orbit.

- Shown here is a panel of relevant information as a set of synthetic radiometer data is processed using the ACCURACy framework.

- This panel just allows us to verify that the simulated data is consistent with our expectations.
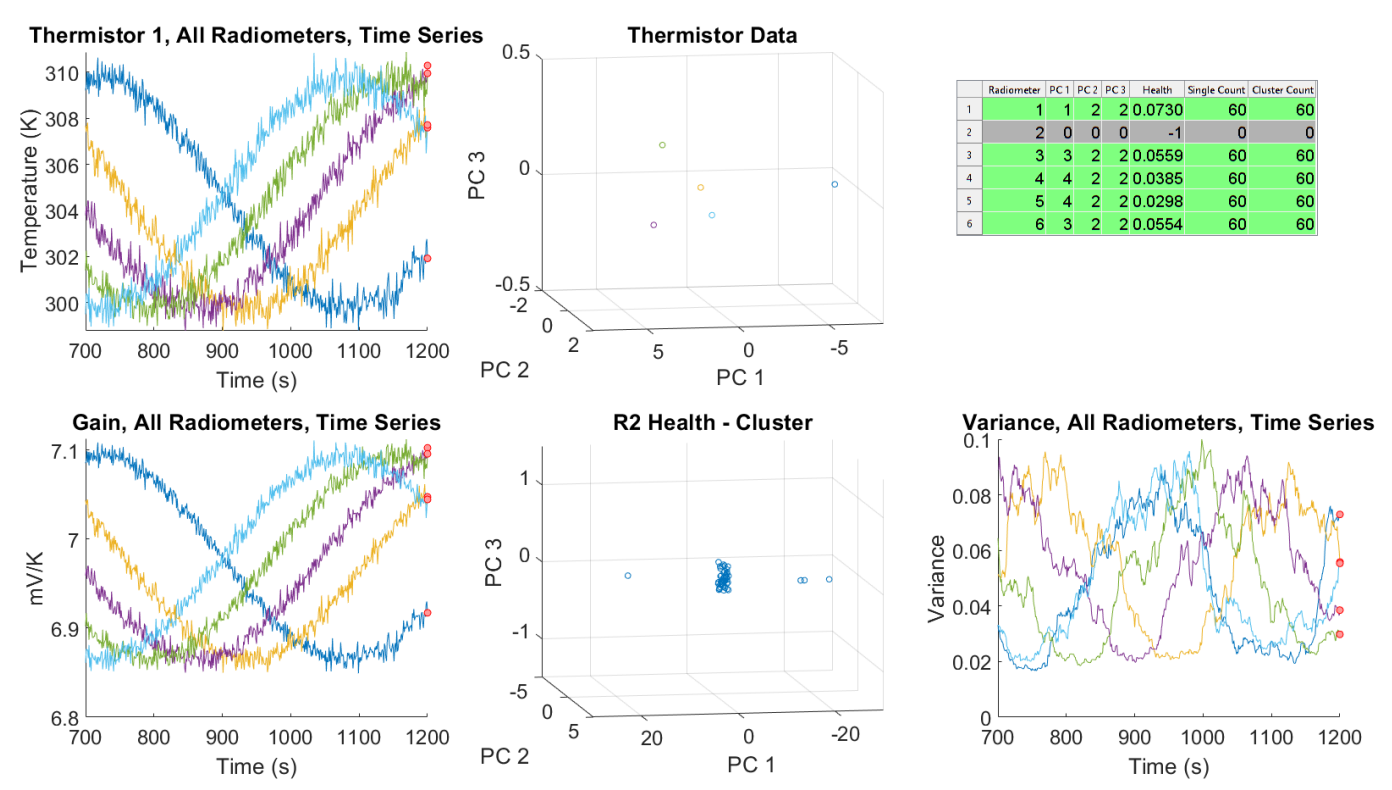

Left to Right, Top to Bottom:

1. Measurements of a single thermistor from each radiometer,

2. Current thermistor data, plotted post-PCA,

3. A table tracking cluster labels and the number of calibration measurements available for each radiometer,

4. Gain plot of each radiometer,

5. A plot showing the health of a single radiometer, defined as a measure of variance,

6. Health of all radiometers, measured as variance of thermistors. 


\section{Simulation}

This simulation consists of 35 CubeSat radiometers orbiting the Earth. Some of the CubeSats are on polar orbits, and some are orbiting close to tropical regions near the equator. This is in part to ensure there are sufficient opportunities for CubeSats to overlap in the simulation.

This simulation can be controlled by adjusting parameters for the radiometers as well as for their orbits.

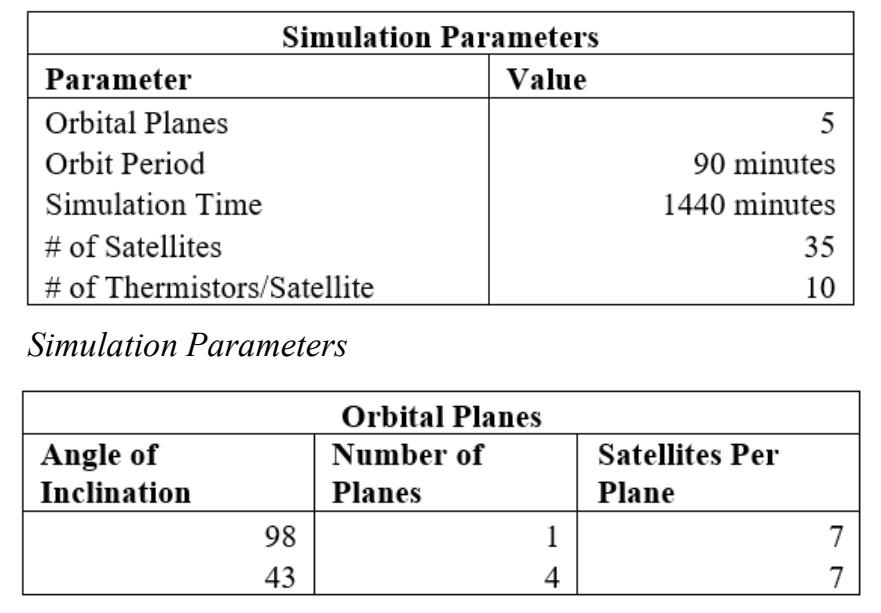

Orbital Planes

\begin{tabular}{|l|r|}
\hline \multicolumn{2}{|c|}{ Calibration Parameters } \\
\hline Parameter & Value \\
\hline Antenna Temperature & $270 \mathrm{~K}$ \\
\# of Calibration Targets & 4 \\
Calibration Temperatures (K) & {$[2.7,210,250,300]$} \\
Receiver Temperature (K) & $1400 \mathrm{~K}$ \\
Bandwidth (GHz) & $2.031 \mathrm{GHz}$ \\
Integration Time (s) & $4.096 \mathrm{~ms}$ \\
\hline
\end{tabular}

Calibration Parameters

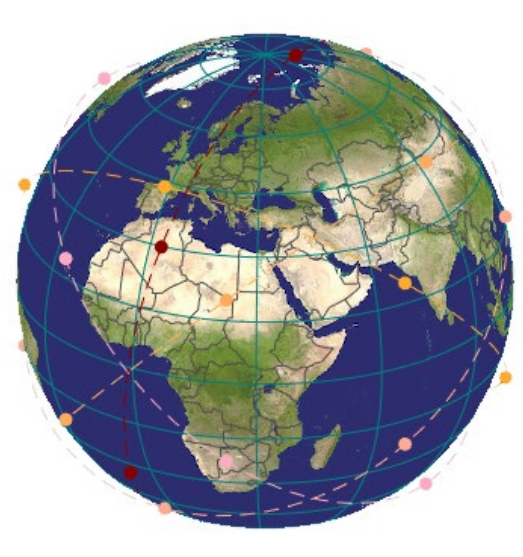




\section{Clustering Analysis}

Each clustering algorithm is evaluated by the quality of the clusters formed using that algorithm.

Four different clustering algorithms investigated for ACCURACy, evaluated using the Mean Distance from Centroids (MDC):

$$
\mathrm{M}_{\mathrm{avg}}=\frac{1}{\mathrm{~N}} \sum_{\mathrm{i}=1}^{\mathrm{k}} \sum_{\mathrm{j}=1}^{\mathrm{n}}\left\|\mathrm{P}_{\mathrm{i}, \text { center }}-\mathrm{P}_{\mathrm{i}, \mathrm{j}}\right\|_{2}
$$

50 seconds of data are plotted along three principal components
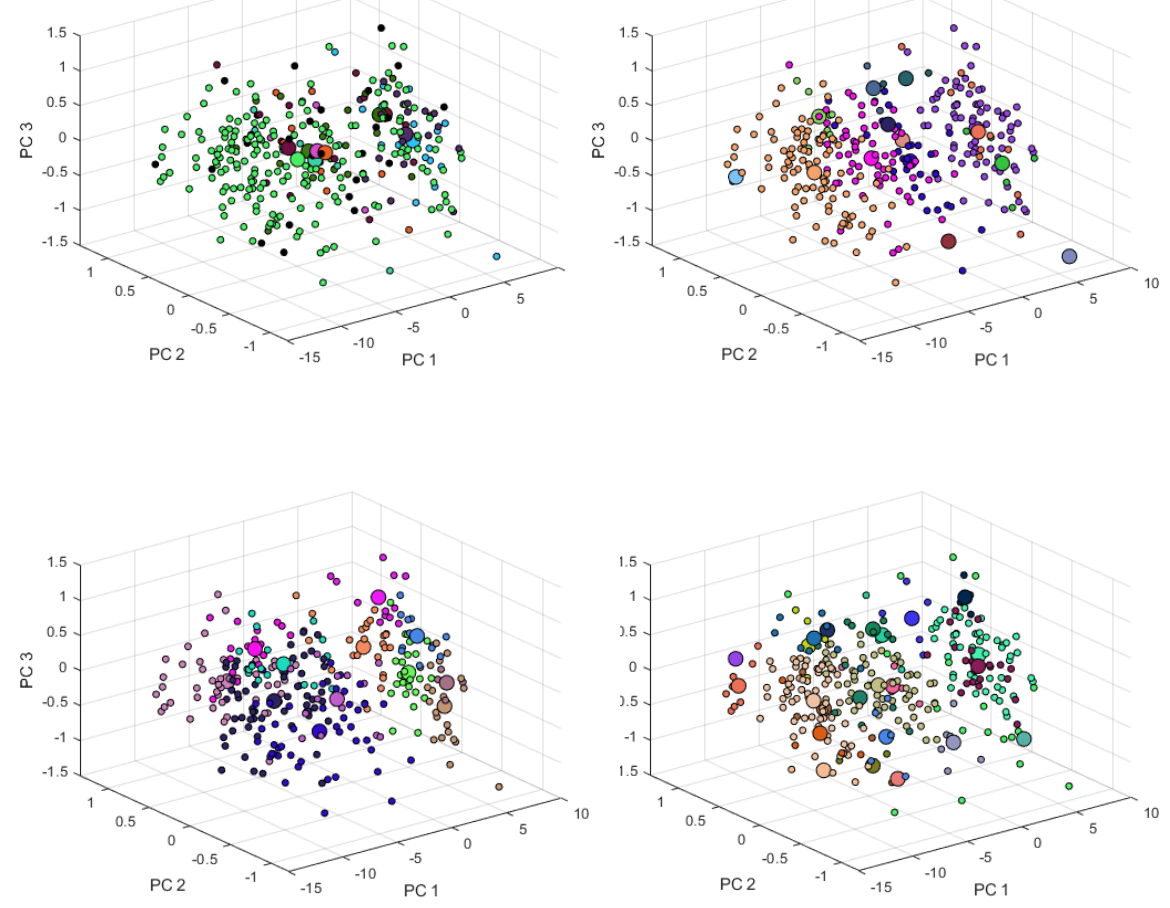

(Top Left) DBSCAN, (Top Right) IDBSCAN, (Bottom Left) Cell-based Clustering, (Bottom Right) Overlapping Cell-based Clustering. Large markers represent cluster centers.

\begin{tabular}{|l|r|}
\hline Clustering Method & \multicolumn{1}{|c|}{ MDC } \\
\hline DBSCAN & 3.3939 \\
\hline Incremental DBSCAN & 0.6849 \\
\hline Overlapping Cell-Based & $\mathbf{0 . 5 1 1 5}$ \\
\hline Normal Cell-Based & 2.1172 \\
\hline
\end{tabular}




\section{Simulation Results}

The simulated constellation of CubeSat radiometers is calibrated using ACCURACy and the SOTA method. A baseline calibration is defined by simulating hot and cold absolute calibration references and performing a 2-point calibration every second, simulating a radiometer operating for $4 \mathrm{~ms}$ every 1 second.

For ACCURACY and the SOTA method, vicarious calibration measurements are obtained according to each method, and re-calibration is performed when sufficient calibration data is available to the system.

RMSE and Standard Deviation values are calculated using $T_{A_{\text {est }}}$ determined by calibrating using ACCURACy, the SOTA overlapping method, and the baseline.

\begin{tabular}{|l|r|r|}
\hline Algorithm & \multicolumn{1}{|c|}{ RMSE } & \multicolumn{1}{c|}{$\boldsymbol{\sigma}^{\mathbf{2}}$} \\
\hline Baseline & 0.93 & 0.84 \\
\hline ACCURACy & $\mathbf{0 . 7 5}$ & $\mathbf{0 . 3 7}$ \\
\hline Conventional SOTA & 1.03 & 0.89 \\
\hline
\end{tabular}
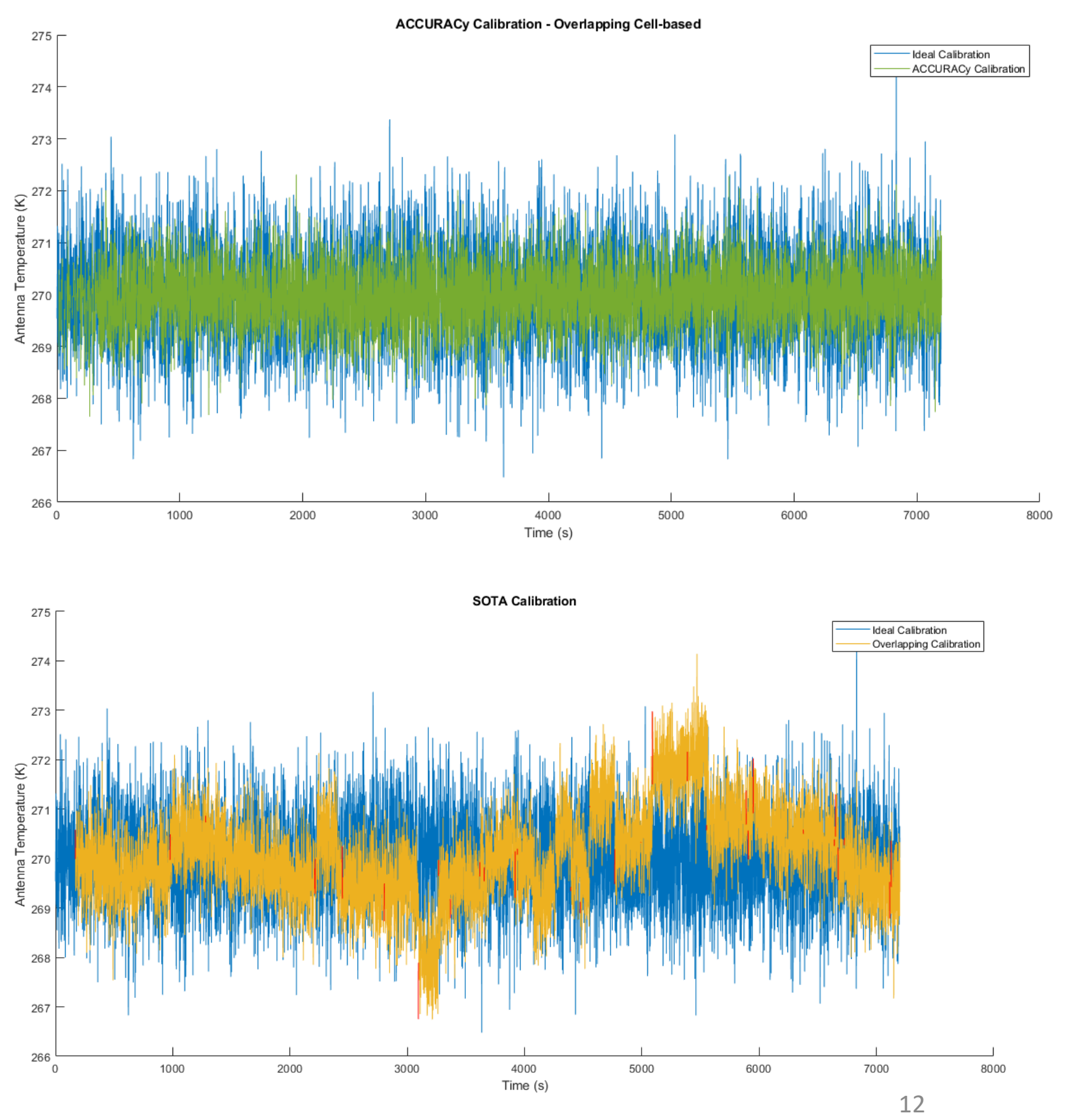


\section{Simulation Results}

Shown are the results of pooling calibration measurements using ACCURACy, with the calibration pools for one instrument plotted as it moves across the surface of the Earth

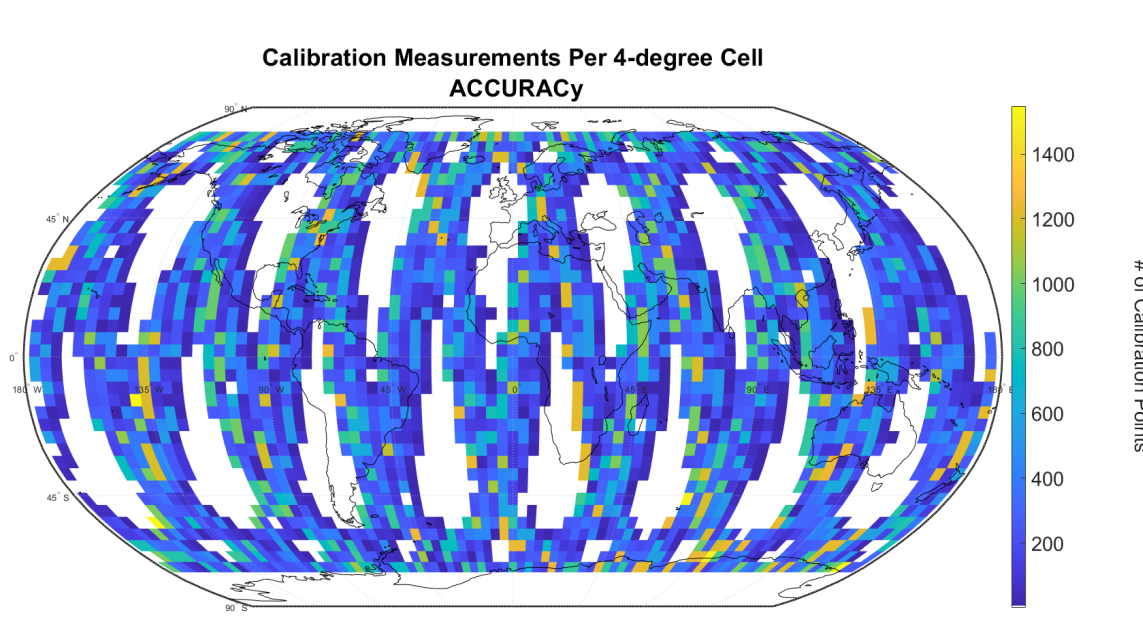

(TOP) After the full simulation using ACCURACy, one satellite maintains a high amount of calibration data to recalibrate frequently along its entire path thus far after 4 orbits

(BOTTOM) Using the SOTA intercalibration is not sufficient for real-time calibration, especially compared to ACCURACy, the entire constellation calibrates very infrequently using the same simulation as ACCURACy on the left.

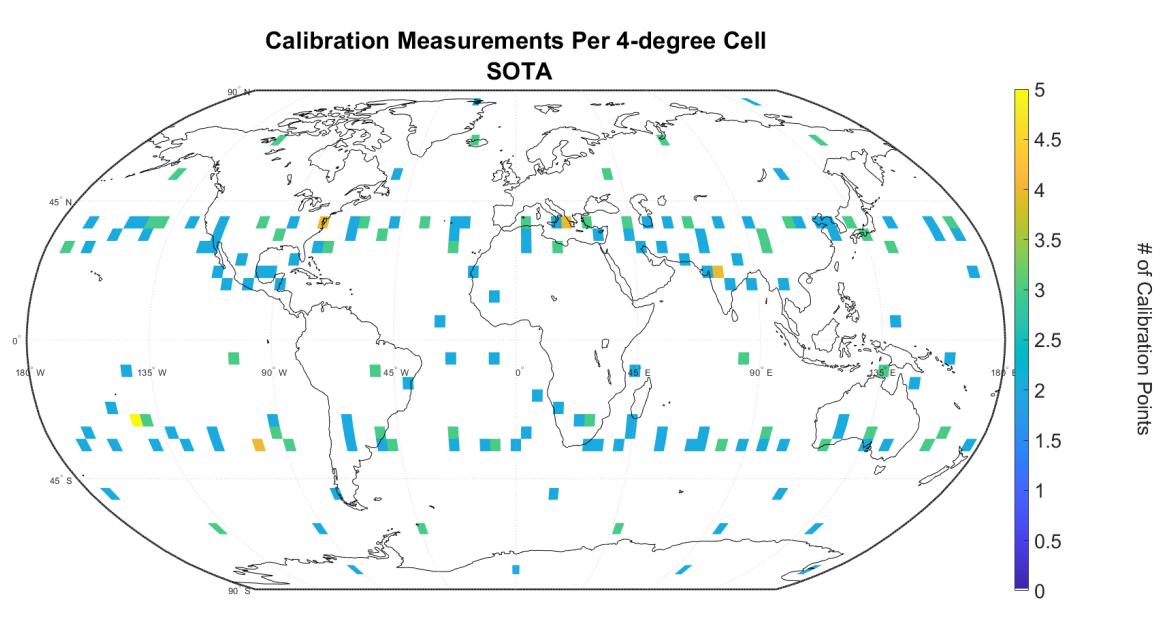




\section{Simulation Results}

(Left) The calculated uncertainty (standard deviation) of the calibrated antenna temperature over 1-minute windows using the baseline, SOTA, and ACCURACY methods.

(Right) A moving mean of the calibrated antenna temperature calculated over a 1-minute window for the baseline, SOTA, and ACCURACy methods.
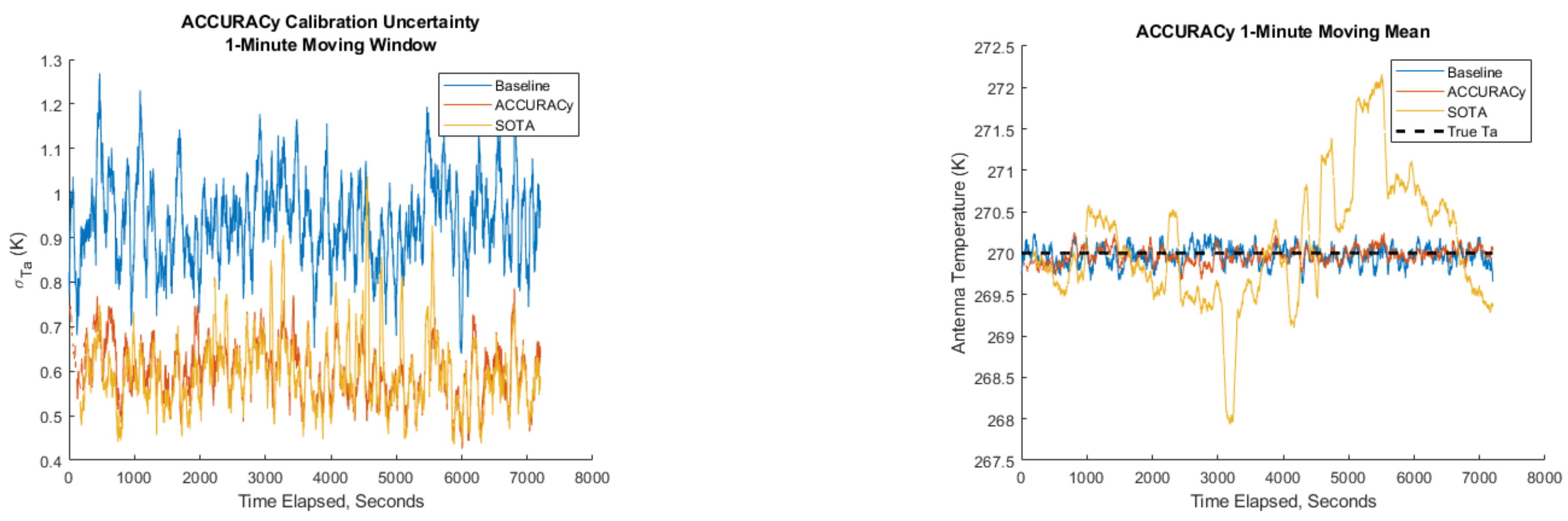


\section{Conclusion}

- The use of ACCURACy to pool calibration measurements and increase calibration data volume and calibration frequency in a real-time scenario is significant compared to the conventional method. In initial simulations, ACCURACy produced a more accurate and precise estimate of antenna temperatures than the conventional SOTA method. 


\section{Future Work}

- Further exploration of more intelligent calibration algorithms will be investigated, including implementing $\mathrm{N}>2$-point adaptive absolute calibration using the input from the Calibration Pool module to reduce and quantify the errors and uncertainties in the calibrated products, as well as the use of other ML algorithms and calibration techniques using neural networks.

- Further plans also include testing ACCURACy using real radiometer data, including plans to acquire and use calibration data from the TROPICS* mission.

- Some things still need to be investigated, including the study of data processing and transmission requirements, scalability, and efficient data sharing between constellation members. 


\section{References}

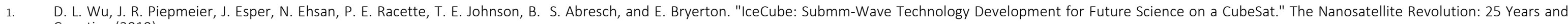
Counting (2019).

Porter, G., "The Global Environmental Monitoring Systems (GEMS) Constellation of Passive Microwave Satellite", 2020.

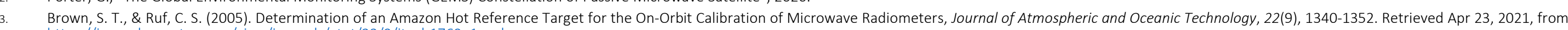
https://journals.ametsoc.org/view/journals/atot/22/9/itech1769 1.xml

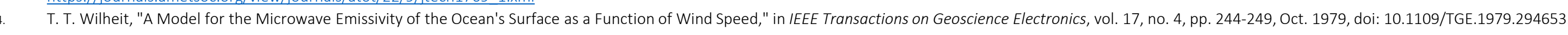

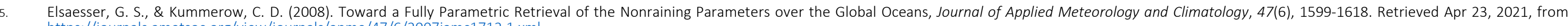
https://iournals.ametsoc.org/view/journals/apme/47/6/2007jamc1712.1.xm/

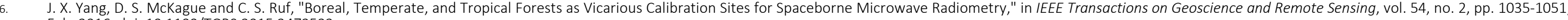
Feb. 2016, doi: 10.1109/TGRS.2015.2472532.

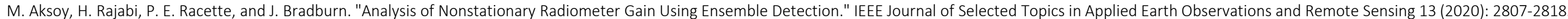

M. Aksoy and P. E. Racette. "A Preliminary Study of Three-Point Onboard External Calibration for Tracking Radiometric Stability and Accuracy." Remote Sensing 11, no. 23 (2019): 2790.

J. Hollinger, Ed., DMSP Special Sensor Microwave Imager Calibration/Validation-Final Report. Washington, DC: Naval Res.Lab., May 1991.

J. P. Hollinger, J. L. Peirce and G. A. Poe, "SSM/I instrument evaluation," in IEEE Transactions on Geoscience and Remote Sensing, vol. 28, no. 5, pp. 781-790, Sept. 1990, doi: 10.1109/36.58964.

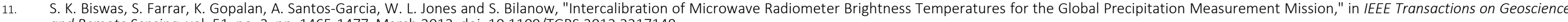
and Remote Sensing, vol. 51, no. 3, pp. 1465-1477, March 2013, doi: 10.1109/TGRS.2012.2217148.

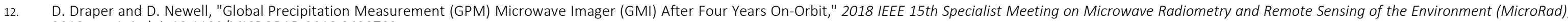
2018, pp. 1-4, doi: 10.1109/MICRORAD.2018.8430702.

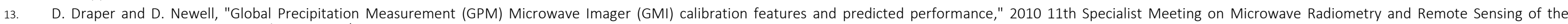
D. Draper and D. Newell, "Global Precipitation Measurement (GPM) M
Environment, 2010, pp. 236-240, doi: 10.1109/MICRORAD.2010.5559555

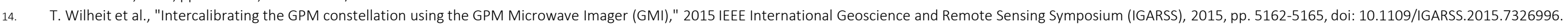

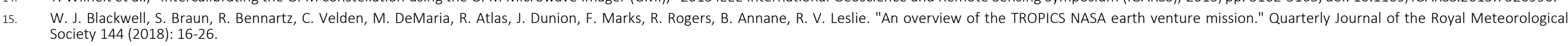

6. M. Artac, M. Jogan, and A. Leonardis. "Incremental PCA for on-line visual learning and recognition." In Object recognition supported by user interaction for service robots, vol. 3, pp. 781-784. IEEE, 2002.

17. S. Chakraborty and N. K. Nagwani. "Analysis and study of Incremental DBSCAN clustering algorithm." arXiv preprint arXiv:1406.4754 (2014).

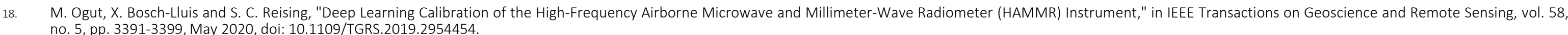

19. T. McClanahan, P. E. Racette, J. Bradburn, private communication, 2020 


\section{Thanks for Listening!}

Contact Information:

Mustafa Aksoy, PI

maksoy@albany.edu

John Bradburn, Graduate Research Assistant

jbradburn@albany.edu 